\title{
Philosophiques
}

\section{Vérité sans vérités ? Réponse à Kevin Mulligan}

\section{Wolfgang Künne}

Volume 38, numéro 1, printemps 2011

Les conférences Hugues Leblanc 2010

URI : https://id.erudit.org/iderudit/1005723ar

DOI : https://doi.org/10.7202/1005723ar

Aller au sommaire du numéro

Éditeur(s)

Société de philosophie du Québec

ISSN

0316-2923 (imprimé)

1492-1391 (numérique)

Découvrir la revue

Citer cet article

Künne, W. (2011). Vérité sans vérités ? Réponse à Kevin Mulligan. Philosophiques, 38(1), 195-217. https://doi.org/10.7202/1005723ar

\section{Résumé de l'article}

Des philosophes de la logique comme Prior et Mulligan considèrent le connecteur de vérité 'Il est vrai que ()' comme étant plus fondamental que le prédicat de vérité '( ) est vrai'. Des philosophes comme Bolzano et Horwich ont adopté l'ordre inverse de priorités et je me suis rallié à eux dans Conceptions of Truth. Je continue à penser que le prédicat " porte la culotte » et vais tenter de désamorcer les arguments contre cette conception, mais je vais aussi rejeter un de mes arguments antérieurs en faveur de celle-ci. d'utilisation que vous pouvez consulter en ligne.

https://apropos.erudit.org/fr/usagers/politique-dutilisation/ 


\title{
Vérité sans vérités? Réponse à Kevin Mulligan
}

\author{
WOLFGANG KÜNNE \\ Université de Hambourg
}

\begin{abstract}
RÉSUMÉ. - Des philosophes de la logique comme Prior et Mulligan considèrent le connecteur de vérité 'll est vrai que ( )' comme étant plus fondamental que le prédicat de vérité '( ) est vrai'. Des philosophes comme Bolzano et Horwich ont adopté l'ordre inverse de priorités et je me suis rallié à eux dans Conceptions of Truth. Je continue à penser que le prédicat «porte la culotte» et vais tenter de désamorcer les arguments contre cette conception, mais je vais aussi rejeter un de mes arguments antérieurs en faveur de celle-ci.
\end{abstract}

\begin{abstract}
Logical Philosophers like Prior and Mulligan regard the truth connective 'It is true that ( )' as more basic than the truth predicate ' $(\quad$ ) is true'. Philosophers like Bolzano and Horwich have adopted the opposite priority setting, and in Conceptions of Truth I joined them. I still think that the predicate 'wears the trousers', and I shall try to defuse the arguments against this view, but I shall also reject one of my earlier arguments for it.
\end{abstract}

\section{Qui porte la culotte (si quelqu'un la porte)?}

Le cadre (frame) «( ) est vrai» est un opérateur servant à former des phrases qui s'applique à des termes singuliers, c'est un prédicat de vérité: en insérant "la conviction la plus profonde d'Alfred", "le logicisme» ou "ceci ", on obtient une phrase. Par contraste, le cadre «il est vrai que ( )" n'autorise pas de remplissement semblable. Il ressemble à «( ) ) en ce qu'il est un opérateur servant à former des phrases qui s'applique à des phrases, c'est un connecteur de vérité: en insérant «la neige est blanche» et «l'arithmétique est réductible à la logique », on obtient des phrases comme résultat ${ }^{1}$. Dans Conceptions of Truth, j'ai brièvement considéré la question de savoir si une de ces deux locutions, prédicat ou connecteur de vérité, était plus fondamentale que l'autre, et, le cas échéant, laquelle était la plus fondamentale. Kevin Mulligan a étudié la question en détail². Selon lui, cette question sur le couple prédicat/connecteur, posée trop peu souvent, fait écho à une question très souvent posée eu égard à d'autres couples: Qui porte la culotte? De mon point de vue,

(CV) Il est vrai que la neige est blanche

n'est qu'une variante stylistique de

1. En caractérisant le deuxième cadre de "connecteur de vérité », je me plie avec réticence à la pratique curieuse des logiciens de qualifier de connecteur un opérateur servant à former des phrases et appliqué à des phrases même si celui-ci ne fait absolument aucune connexion.

2. Dans le présent article, la discussion des thèses de Mulligan se réfère toujours à Mulligan 2010, un commentaire sur mon livre Conceptions of Truth (N.d.T.). 
(PV) Que la neige est blanche est vrai

et $(\mathrm{PV})$ est à

$(\mathrm{PV}+)$ La proposition que la neige est blanche est vraie

ce que «Sept est premier» est à «Le nombre sept est premier». Pour tenter de clarifier notre concept usuel de vérité, il est préférable de nous concentrer sur le prédicat de vérité immanquable dans (PV+) et identifiable dans $(\mathrm{CV})$. La proposition exprimée par (CV) est de manière non évidente ce que la proposition exprimée par $(\mathrm{PV}+)$ est de manière évidente: une proposition dans laquelle quelque chose est classifié comme vrai. En ce sens, je plaide en faveur de l'option du prédicat. Mulligan trouve que mon choix de priorités n'est pas plausible. Selon lui, il n'y a que la blancheur qui est prédiquée dans $(\mathrm{CV})$. Pour tenter de clarifier notre concept usuel de vérité, il serait préférable à son avis de nous concentrer sur le connecteur de vérité. C'est alors la locution qui porte la culotte: au fond, selon lui, parler de la vérité ne requiert aucune vérité (et aucune fausseté).

En ce qui concerne son point principal, Mulligan a un précurseur proéminent. Arthur N. Prior écrivait: "Les mots "fait" et "vérité" sont dans leur usage primaire des parties inséparables des phrases adverbiales [...] "c'est un fait que" et "il est vrai que" 3 ." Dans cette remarque, Prior anticipe également un aspect de la comparaison éclairante de Mulligan entre le discours sur la vérité et celui sur les faits: au fond, ce dernier ne nécessite aucun fait.

Mulligan expose ensuite une «conception » qu'il considère avec raison incompatible avec la mienne. Je vais ici faire référence à cette conception comme étant la sienne, à tort ou à raison:

1. $\mathrm{p}$

2. il est vrai que $\mathrm{p}$

3. que p est vrai

4. la proposition que $\mathrm{p}$ est vrai

5. (1) ssi (2) ssi (3) ssi (4)

6. si (2), alors (2) parce que (1)

7. si (3), alors (3) parce que (1)

8. si (4), alors (4) parce que (1)

9. $\quad$ si (3), alors (3) parce que (2)

10. si (4), alors (4) parce que (3)

Selon la conception de Mulligan, (9) affirme que c'est le connecteur de vérité qui porte la culotte eu égard à un type de prédication de vérité; (10), pris avec (9), affirment la même chose à propos d'un autre type de prédica-

3. Prior, 1967, p. 229 (un des meilleurs articles encyclopédiques jamais écrits). 
tion de vérité. Le morphème de vérité interviendrait comme prédication à la fois dans (3) et (4), et dans chaque cas il prendrait une expression nominale pour faire une phrase. Mais dans (2), le morphème de vérité intervient comme connecteur. Selon Mulligan, les séquences formées par des instances de «que $p$ » interviennent comme des expressions nominales dans des instances de (3), alors que dans des instances de (2), elles interviennent comme de simples fragments de phrases ${ }^{4}$.

En ce qui concerne la variété des façons de parler de la vérité, j'endosse l'ensemble de biconditionnelles comme "la neige est blanche ssi (CV) ssi (PV) ssi (PV+) », qui sont représentées schématiquement par la ligne (5) dans le schéma de Mulligan ${ }^{5}$, et je suis aussi d'accord avec la plupart des «énoncés explicatifs » schématiques qui s'en suivent. Intuitivement, la proposition que la neige est blanche tire sa vérité de l'être blanc de la neige, et:

(8) Si la proposition que $p$ est vraie, alors la proposition est vraie parce que $p$

est la manière préférée par Bolzano et moi-même pour expliciter ce genre d'intuition (Conceptions of Truth, p. 150-157). C'est ce que Mulligan appelle "la vérifaction sans vérifacteurs ${ }^{6}$. Intuitivement, c'est en vertu de l'être blanc de la neige qu'il est vrai que la neige est blanche, et que la neige est blanche est vraie en raison de l'être blanc de la neige. Je suis donc aussi d'accord pour endosser (6), «s'il est vrai que $p$, alors (il est vrai que $p$ ) parce que $p$ » et (7), «Si que $p$ est vrai, alors que $p$ est vrai parce que $p{ }^{7}$. Mais qu'en est-il des deux derniers schémas explicatifs de Mulligan?

(9) Si que $p$ est vrai, alors que $p$ est vrai parce qu'il est vrai que $p$

(10) Si la proposition que $p$ est vraie, alors cette proposition est vraie parce que $p$ est vrai.

Mulligan commente: "(9) affirme que le connecteur de vérité porte la culotte en ce qui concerne un type de prédication de vérité; (10) pris avec (9) affirme la même chose pour un autre type de prédication de vérité ». Il considère "la proposition que $p$ est vraie" comme plus exigeante conceptuellement que "que $p$ est vrai", et je suis d'accord sur ce point. Dans Conceptions of Truth, p. 254-55, j'ai comparé (PV+) avec «le nombre sept est premier». Dans les deux phrases, il y a un terme général qui exprime un concept pour lequel la place est libre lorsqu'on comprend ses contreparties démaquillées, $(\mathrm{PV})$ et «sept est premier». Mais on peut comprendre la dernière phrase

4. Passage adapté de Mulligan 2010, p. 567 (N.d.T.).

5. En supposant qu'elles n'engendrent pas de paradoxes. Nous verrons que certaines de ces biconditionnelles posent des problèmes pour la conception de Mulligan.

6. Cf. Schnieder, 2006.

7. J'ai pris la liberté d'insérer des parenthèses pour éviter une ambiguïté syntaxique. Comme nous allons le voir, Mulligan se met dans une position inconfortable face aux antécédents dans ses schémas (7) et (9). 
sans avoir encore fait l'acquisition des concepts généraux qui sont exprimés dans la première phrase. Dans les deux cas, la phrase verbeuse n'est pas un synonyme de sa contrepartie économe, et, dans les deux cas, il semble correct de dire que la vérité de ce qui est exprimé par la phrase plus longue est en partie redevable de la vérité de ce qu'exprime la phrase plus courte. Car, généralement, si le $\mathrm{G} a$ est $F$, alors le $\mathrm{G} a$ est $F$ parce que ( $a$ est $F \& a$ est un $G$ ); donc si le $\mathrm{G} a$ est $F$, alors le $\mathrm{G} a$ est $F$ en partie parce que $a$ est $F$. Comprise de cette manière, la ligne (10) de Mulligan est plausible.

Mais la ligne (9) ne me semble pas plausible du tout. Selon Mulligan, (PV) est conceptuellement plus exigeant que (CV), et, en l'affirmant avec une force assertorique, on accepte un engagement ontologique que l'on n'accepterait pas en affirmant $(\mathrm{CV})$ de manière assertive. Pour autant que je sache, en latin classique, $(\mathrm{CV})$ et $(\mathrm{PV})$ devraient être formulées par "verum est + + "nivem albam esse», et en raison de la construction accusatif-cuminfinitif, la traduction ne permet pas un parsage du connecteur de vérité 8 . Supposons que j'aie raison en ce qui concerne les Romains. Si c'est le cas, dans leur langage, l'énoncé supposément explicatif qui instancie (9) se révèle alors être une fausseté éclatante, car, comme le dit Mulligan, des instances de « $p$ parce que $p$ » expriment des faussetés si (mon ajout) les deux occurrences de la même phrase expriment la même proposition ${ }^{9}$. Les locuteurs du latin classique sont-ils contraints à accepter un engagement ontologique que les locuteurs de l'anglais peuvent aisément éviter lorsqu'il est question de la vérité? Je ne crois pas que ce soit une question raisonnable. Mais laissons les Romains à leur destinée. Supposons que Anne et Ben regardent des photos de leurs anciens camarades de classe. Anne affirme que Tom et Dick étaient tous deux très ennuyeux, mais Ben proteste, en pointant d'abord vers la photo de Tom, puis ensuite vers celle de Dick: "C'est vrai qu'il était ennuyeux, mais que lui était ennuyeux, ce n'est pas du tout vrai. » Je ne vois pas en quoi on aurait besoin d'un appareillage conceptuel plus élaboré (autre que la maîtrise du concept de négation) pour comprendre la seconde moitié de l'énoncé de Ben que pour en comprendre la première moitié, ni

8. La construction «verum est» + «quod nix alba est» est disponible seulement en latin médiéval, cf. Kretzmann, 1970, p. 776.

9. Schnieder (à paraître -2) offre des exemples qui montrent la nécessité d'une telle clause. Qu'une instance de " $p$ parce que $p$ " puisse exprimer une vérité, cela est parfois dû à une ambiguiité lexicale ou à la sensitivité au contexte, comme c'est le cas dans "l'hôte [inviée] $_{\text {a fait }}$ un scandale parce que l'hôte [maitre de maison] a fait un scandale" et dans "cet hôte a fait un scandale parce que cet hôte a fait un scandale». [Si on remplace "parce que» par "si », on voit que l'affirmation selon laquelle toutes les instances de "si $p$ alors $p$ » expriment des vérités logiques nécessite elle aussi la même clause. Cf. Künne 2009, p. 300-303.] Parfois, une instance de " $p$ parce que $p$ " peut exprimer une vérité parce qu'il y a une interprétation selon laquelle elle n'est pas composée de deux phrases complètes. "La plupart des gens célèbrent Noël parce que la plupart des gens célèbrent Noël ", disait le satiriste Kurt Tucholsky. Il n'a raison que si on construit sa remarque de la manière suivante: «La plupart des gens, $x$, sont tels qu'ils célèbrent Noël parce que la plupart des gens autres que $x$ célèbrent Noël.» 
comment il pourrait y avoir des "différences d'engagement ontologique» entre les deux moitiés de sa réponse. Peut-on accepter un engagement ontologique et s'en départir, simplement en ajoutant ou en enlevant un pronom postiche et en changeant l'ordre des mots dans le médium de l'assertion? Une telle variation n'est-elle pas trop fragile pour porter un tel fardeau?

Comme Mulligan le souligne, le connecteur de vérité est itérable alors que le prédicat de vérité ne l'est pas: des instances de *(la proposition) que $p$ est vrai est vraie*, etc., violent en effet les lois de la grammaire, alors que des instances de «il est vrai qu'il est vrai que $p$ ", etc., ne le font pas. Mais il est tout aussi remarquable qu'il y a une série de prédications de vérités emboîtées qui est logiquement équivalente avec la série des itérations «il est vrai que ": on peut emboîter toute attribution de vérité dans le cadre «la proposition que ... est vraie» - la phrase «la proposition que $p$ est vraie» étant une d'entre elles. (Si on laisse tomber le préfixe «la proposition», le résultat n'est plus grammatical.)

Mulligan défend la thèse que dans la construction «il est vrai que $p$ ", les instances de "que $p$ » n'interviennent pas comme "expressions nominales » ou comme " unités nominales », que les «instances de "il est vrai que $p »$ ne contiennent aucune nominalisation ». Prima facie, cette thèse semble absolument paradoxale: que peut bien être "que la neige est blanche" si ce n'est pas une nominalisation de la phrase «la neige est blanche »? Mais si on adopte la perspective de Mulligan, l'impression de paradoxalité disparaît. Si le parsage de $(\mathrm{CV})$ en connecteur de vérité est le seul parsage correct de cette construction, alors celui-ci contient seulement une occurrence inauthentique de la subordonnée complétive "que la neige est blanche", puisque le mot "que» appartient au connecteur. Prenons une autre question rhétorique: que peut bien être «Napoléon était un grand général» si ce n'est pas une phrase? Ce ne sont pas toutes les occurrences de cette séquence de mots qui sont des phrases. Par exemple, son occurrence dans $(S)$ "L'homme qui a vaincu Napoléon était un grand général» n'est pas une occurrence authentique de la phrase "Napoléon était un grand général », car dans $(S)$, «Napoléon" est une partie du terme singulier complexe en position sujet. Or comme Peter Geach, à qui j'emprunte cet exemple, l'a formulé de manière caractéristique, "même une grammaire scolaire superficielle» confirmerait que la séquence de mots constituée de "Napoléon» et "était un grand général» n'intervient pas dans $(S)$ en tant que phrase ${ }^{10}$. Mais bon nombre de grammairiens superficiels, et Mulligan serait le premier à l'admettre, seraient prêts à soutenir que la séquence composée de «que» et «la neige est blanche » intervient effectivement dans $(\mathrm{CV})$ comme subordonnée complétive. Et bien sûr je suis moi-même prêt à soutenir cela, car je ne considère pas que le parsage de telles phrases en connecteur de vérité soit obligatoire. Bien plus, je considère le parsage en prédicat de vérité non seulement comme

10. Geach, 1972, p. 110; cf. également p. 229. 
étant tout aussi acceptable, mais même comme étant structurellement plus révélateur que son compétiteur. On ne peut sérieusement nier qu'il arrive au moins à l'occasion que les parsages en connecteur de vérité et en prédicat de vérité soient également acceptables. Après tout, on peut découvrir des connecteurs de vérité aussi dans (PV) et dans (PV+), nommément "que ( ) est vrai» et «la proposition que ( ) est vraie ». Les occurrences de la phrase «la neige est blanche» dans (PV) et dans (PV+) sont certainement authentiques. Et $i c i$, Mulligan serait d'accord avec moi sur le fait que le parsage en prédicat de vérité est non seulement tout aussi acceptable mais aussi structurellement plus révélateur.

Mulligan écrit: "Si [(PV) et (CV)] veulent dire la même chose ou sont synonymes, alors le résultat de la combinaison des deux avec "parce que" serait fausse ${ }^{11}$." Comme je l'ai dit il y a un moment, j'accepte à la fois l'antécédent et le conséquent. Mais je rejette la conditionnelle, car je pense qu'une instance de " $p$ parce que $q$ » peut exprimer une vérité même si les phrases enchâssées veulent dire la même chose. Prenez mon exemple du «parce que» de l'explication causale dans Conceptions of Truth, p. 155: «Il est ton cousin de premier degré parce qu'il est l'enfant du frère ou de la sœur de tes parents. » Ici, les phrases à gauche et à droite du «parce que» ont «la même signification, dans un sens large du mot", et pourtant une énonciation de toute la phrase peut très bien exprimer une vérité. L'observation selon laquelle, dans les cas standard, on ne peut dire quelque chose de vrai avec une instance de " $p$ parce que $p$ » ne supporte pas l'exigence de la différence de signification, puisque l'exemple que je viens de présenter n'est pas, de toute évidence, une instance de ce schéma répétitif. Néanmoins, il y a ici un problème. Si l'explanandum et l'explanans dans notre explication conceptuelle expriment la même proposition, notre affirmation "parce que» semble entrer en conflit avec le principe très plausible selon lequel toute explication est irréflexive: pour tout $x$, ce n'est pas le cas que $x$ explique $x$. Si nous ne voulons nier que l'explanandum et l'explanans expriment la même proposition ${ }^{12}$ dans une explication conceptuelle, nous devrions dire: une phrase de la forme " $p$ parce que $q$ ", dans laquelle les phrases enchâssées expriment la même proposition, ne peut exprimer une vérité à moins que ces phrases diffèrent quant à leur forme logique. Si cette condition nécessaire est remplie, alors les propositions enchâssées articulent la même proposition de différentes manières. Frege est proche de cette idée de l'articulation lorsqu'il dit de deux phrases que l'une "exprime le même

11. Cf. Mulligan, 2010, $\mathbb{} 4$.

12. Dans Conceptions of Truth, p. 369-371, j’ai donné des raisons inspirées par Mates pour nier cette thèse. 
sens» que l'autre, "mais de manière différente» (drückt zwar denselben Sinn aus..., aber in anderer Weise $)^{13}$.

Mais tournons-nous maintenant vers les questions de Mulligan concernant ma conception ainsi que ses objections.

\section{L'erreur du Canard et la question de la généralisabilité}

Puisque j'ai attribué une référence cataphorique ${ }^{14}$, et donc une référence au «il» (it) dans «il est vrai que $p$ » (It is true that $\mathrm{p}$ ), je suis un peu embarrassé de me trouver dans un rôle semblable à celui du Canard de Lewis Carroll:

[La Souris continua:]

- Stigand, l'archevêque patriote de Cantorbery, trouva cela $(i t)$.

- Trouva quoi? dit le Canard.

- Il trouva cela, répondit la Souris avec impatience. Assurément vous savez ce que «cela» veut dire.

- Je sais parfaitement ce que «cela» veut dire; par exemple: quand j'ai trouvé cela bon, «cela» veut dire un ver ou une grenouille, ajouta le Canard. Mais il s'agit de savoir ce que l'archevêque trouva.

La Souris, sans prendre garde à cette question, se hâta de continuer.

— L'archevêque trouva cela de bonne politique d'aller avec Edgar Atheling à la rencontre de Guillaume, pour lui offrir la couronne $[\ldots]^{15}$.

La question n'est ni celle de savoir ce que l'archevêque trouva, ni celle de savoir à quoi «il» réfère dans «il est vrai que $p$ ». Mulligan a raison contre Horwich et Künne: il n'y a pas ici de référence, qu'elle soit cataphorique ou non. On peut mener son point à bon port à l'aide d'une comparaison. Au tout début du Guillaume Tell de Schiller, un jeune pêcheur chante sur son bateau:

(Sch) Es lächelt der See, er ladet zum Bade ${ }^{16}$.

Que font ici les pronoms? Le «er» dans la seconde phrase est anaphorique, il désigne ce que son «antécédent» («der See») désigne dans la chanson du jeune pêcheur, le Lac des Quatre-Cantons. Par contraste, le

13. Frege, 1891, p. 11. Je commente ce passage dans Künne, 2007, où j'introduis l'idée d' "articuler la même proposition de différentes manières». Schnieder (à paraître-1) plaide en faveur de l'application d'une idée plutôt semblable au «parce que» de l'explication conceptuelle.

14. Pour ceux qui ne sont pas familiarisés avec cette terminologie, prenez par exemple l'emploi du «elle» dans (1) «si Anne a le temps, elle se joindra à nous » et (2) «si elle a le temps, Anne se joindra à nous». Dans (1), nous avons une anaphore, dans (2) une cataphore.

15. Lewis Carroll, Aventures d'Alice aux pays des merveilles, trad. fr. Henri Bué, Londres, MacMillan 1869, p. 32-33 (N.d.T.).

16. Traduction française, inutile pour mon ami presque Suisse: «L'onde semble sourire, elle invite à se rafraîchir dans son sein! » trad. fr. D’Aubigner, p. 37. 
«es» dans la première phrase est explétif, c'est un pronom postiche: il ne désigne rien, il est vide sémantiquement ${ }^{17}$. Dans Conceptions of Truth, mon erreur ressemble à celle de confondre la première phrase dans (Sch) avec "Er lächelt, der See». Ici, le pronom (notons bien le genre!) est effectivement cataphorique: il reçoit son contenu sémantique de son subséquent. Mais gardons-nous de ne pas voir la forêt avant les arbres: peu importe si c'est la phrase de Schiller qui est employée ou encore sa contrepartie cataphorique, le contenu de l'énoncé complet demeure le même, et il est identique avec la proposition exprimée dans le même contexte par une phrase dont la substitution ruinerait le rythme de la chanson: "Der See lächelt».

Bolzano et Horwich sont convaincus que $(\mathrm{CV})$, "Il est vrai que la neige est blanche ", exprime la même proposition que (PV), "Que la neige est blanche est vrai ", mais, contrairement à ce que j'ai insinué dans mon livre, la réponse que mon premier témoin (qui est aussi mon favori) donne à la question de savoir pourquoi c'est ainsi diffère de celle donnée par Horwich. Bolzano considère les instances de «il est vrai que $p$ » comme pléonastiques lorsqu'il déclare que le premier mot est "superflu» ${ }^{18}$. (Certains grammairiens caractérisent les explétifs de "pronoms pléonastiques".) Si la partie X d'une phrase $S$ est propositionnellement superflue, alors les mots dont $\mathrm{S}$ est constituée moins $\mathrm{X}$ expriment ce qui est exprimé de manière peu économique par S. En ce sens, le mot «vraiment» dans "Socrate était vraiment sage » est superflu ${ }^{19}$. Il peut parfois arriver qu'en retirant l'expression superflue il faille changer l'ordre original des mots, comme c'est le cas de la première phrase de (Sch). Aux yeux de Bolzano, la transition de (CV) à (PV) est une transition du même genre que celle allant de la phrase de Schiller à «Der See lächelt». Cela me semble parfaitement juste.

Je ne veux pas nier par là qu'il y a aussi des différences significatives entre les deux cas. Par définition, les pronoms explétifs ne désignent rien, mais il y a explétif et explétif. Le "es" dans $(\mathrm{Sch})$ tend à disparaître lorsque (Sch) est nié, et il disparaît lorsque (Sch) est transformé en une phrase interrogative de type oui/non. De plus, il n'a aucune contrepartie dans la traduction française de $(\mathrm{Sch})$. Le «il» dans $(\mathrm{CV})$ demeure là, même lorsque $(\mathrm{CV})$

17. Les Lieder et les poèmes allemands abondent de telles constructions. "Es ist ein Ros' entsprungen" (chant de Noël), "Es schlug mein Herz, geschwind zu Pferde!" (Goethe), "Es bellen die Hunde, es rasseln die Ketten" (Müller/Schubert), "Es klappert die Mühle am rauschenden Bach» (Volkslied) [...]. Je perçois une similarité avec le pronom dans la chanson irlandaise de régiment "It's a long way to Tipperary", qui semble vouloir dire la même chose que «The way to Tipperary is long», mais évidemment, un Mulligan est ici bien mieux placé pour en juger.

18. Bolzano, 1837, vol. II, p. 216, cité par Mulligan en $\$ 5$. Pour Bolzano, «équivalent » (gleichgeltend) signifie extensionnellement équivalent, mais dans le cas discuté il maintient l'identité propositionnelle, comme le suggère fortement dans le texte l'ajout de "complètement ", l'attribution du caractère superfétatoire ainsi que l'exemple suivant.

19. Cf. Bolzano, 1837, vol. I, p. 123, sur «in Wahrheit» et «wirklich». 
est niée ou transformée en une phrase interrogative de type oui/non, et il a une contrepartie dans la traduction allemande de $(\mathrm{CV})$.

Quoi qu'il en soit, je persiste à soutenir que l'analogia proportionalitatis suivante est valide:

[1] «Il est vrai que $p$ » : [2] «Que $p$ est vrai» : :

[3] «Il est vrai, l'énoncé qu'elle a fait » : [4] «L'énoncé qu'elle a fait est vrai»

Indépendamment de la disanalogie concernant le rôle de «il » dans [1] et [3], je persiste à penser que les analogies positives suivantes sont valides: D'abord, si on supprime « il » dans les instances de [1] et [3] et qu'on déplace « est vrai » à la fin, on exprime encore la même proposition. Ensuite, dans les deux cas, la phrase de droite dépeint mieux la structure de la proposition exprimée, structure consistant en une partie exprimée par le prédicat «( ) est vrai » et en une autre exprimée par le terme singulier qui sature ce prédicat. Dans les deux cas, les phrases couplées ont la forme logique d'une prédication, et donc elles ne diffèrent pas plus, eu égard à leur forme logique, que «Tweedledum et Tweedledee sont similaires » et «Tweedledum est similaire à Tweedledee». (Ces deux points renforcent le rejet du schéma explicatif (9) de Mulligan) ${ }^{20}$.

Mulligan indique que non seulement «vrai » peut faire partie d'un prédicat et d'un connecteur, mais aussi "(pas) le cas", "possible", "problable", "certain ", etc. Il se demande si je serais prêt à appliquer mon ordre de priorités aussi à ces expressions qui «semblent être des membres d'une seule et même famille ${ }^{21}$. Je pense que chacun de ces cas devrait être étudié individuellement, et que cela nécessiterait un canevas beaucoup plus large que ce qui est ici disponible. Je vais tenter d'y répondre seulement pour l'expression "pas le cas ». Mais d'abord, je veux suggérer de retirer un intrus dans la liste des membres de la famille de Mulligan. Sa question « la construction en connecteur doit-elle être comprise dans chaque cas comme secondaire eu égard à la construction prédicative correspondante?" s'applique seulement à des constructions du type «il (n')est (pas) $\Phi$ que $p$ » qui ont une contrepartie prédicative "Que $p$ (n')est (pas) $\Phi$ ». Et la même chose vaut pour sa question à savoir si, dans toutes ces constructions, il considère le «il» comme explétif (et non pas comme cataphorique). Mais alors «si $p$ alors $q$ » ne devrait pas être sur la liste de Mulligan, puisqu'ici la construction ne contient pas un "que» suivi par une phrase. Comme les instances de "non $p$ », « $p$ et $q$ » et " $p$ ou $q$ », les conditionnelles n'ont aucun parsage prédicatif, et la question de la priorité ne se pose donc pas. (Il est important de noter en passant que la question se pose eu égard à la déplorable fausse lecture des conditionnelles par Russell et C. I Lewis, à savoir "Que $p$

20. $C$ f. la fin de $\mathbb{1} 1$ plus haut.

21. Cf. Mulligan, 2010, p. 571. 
implique que $q »$ : cette lecture a un parsage en connecteur, "Que ( ) implique que [ ]» et un parsage en prédicat, "( ) implique [ ]»).

Conséquemment, je ne considère pas la locution adverbiale «ne ... pas » dans "la neige n'est pas bleue » et l'opérateur phrastique de négation dans « $\neg(2+2=5)$ » comme étant dans le même bateau que le connecteur verbeux dans des instances de «Il n'est pas le cas que $p$ ». Bien que les victimes de nos cours de logique élémentaire soient rarement averties sur ce point, prononcer « $\neg$ » comme «il n'est pas le cas que» est une fausse lecture, car dans notre langage cette dernière expression n'est pas un connecteur insécable (bien qu'elle contienne un opérateur atomique de négation). Si elle était insécable, nous ne pourrions insérer de parenthèse, comme dans «il n'est pas le cas - nous le savons tous - que $p »^{22}$. (Naturellement, l'objection de la parenthèse peut aussi être soulevée, et elle l'a été par Kent Wilson, contre la thèse selon laquelle le connecteur de vérité est insécable. Je vais revenir sur ce point.)

Je suis prêt à maintenir les analogues de mes affirmations concernant les interrelations entre "la neige est blanche", $(\mathrm{CV}),(\mathrm{PV})$ et $(\mathrm{PV}+)$ pour les contreparties «la neige n'est pas bleue » et "pas le cas» (contreparties en $\mathrm{N}$ ) des phrases en $\mathrm{V}((\mathrm{CV}),(\mathrm{PV})$ et $(\mathrm{PV}+))$ :

(CN) Il n'est pas le cas que la neige est bleue.

(PN) Que la neige est bleue n'est pas le cas.

$(\mathrm{PN}+)$ L'état de choses que le neige est bleu n'est pas le cas (n'a pas de validité).

Mes accords et désaccords avec Mulligan à propos de ces phrases en $\mathrm{N}$ font écho à ceux qui concernent des phrases en $\mathrm{V}$. De un, la neige n'est pas bleue ssi $(\mathrm{CN})$ ssi $(\mathrm{PN})$ ssi $(\mathrm{PN}+)$. De deux, le «il» dans $(\mathrm{CN})$ est un pronom explétif, donc $(\mathrm{CN})$ exprime la même proposition que (PN). De trois, $(\mathrm{PN})$ est à $(\mathrm{PN}+)$ ce que «sept est premier » est à «le nombre sept est premier». Intuitivement, toute phrase en $\mathrm{N}$ est à propos d'exactement la même chose que ce dont il est question dans $(\mathrm{PN}+)$, à savoir un état de choses (et elle est concerne seulement indirectement la neige et l'être bleu). Ainsi, aucune des phrases en $\mathrm{N}$ n'exprime la même proposition que la pleine négation, car la dernière n'est certainement pas à propos d'un état de choses. De quatre, parce que la neige n'est pas bleue, $(\mathrm{CN}),(\mathrm{PN})$ et $(\mathrm{PN}+)$. De cinq, $(\mathrm{PN}+)$ parce que $(\mathrm{PN})$. Et j'ajoute un sixième énoncé parallèle au quatrième:

22. Cf. Künne, 2010, p. 559. L'hypothèse de Mulligan selon laquelle les constructions avec des connecteurs verbeux ( «Il est (...) que $p$ ») sont « dérivées de» contreparties avec des connecteurs à un seul mot ( ( $(.$.$) ment, p$ ») n'est ici d'aucune aide. J'admets qu'on peut insérer une parenthèse entre "probablement» et la phrase qu'il gouverne. Mais s'il y a un connecteur d'un seul mot servant à contredire et qui peut être préfixé à une phrase en anglais, je ne l'ai jamais vu. 
(CN), (PN) et $(\mathrm{PN}+)$ parce que $\neg$ (la neige est bleue). Cela confirme mon affirmation que «Il n'est pas le cas que» est une fausse lecture de « $\neg »^{23}$.

Bien entendu, on peut stipuler que le connecteur verbeux doit être compris comme s'il était aussi peu structuré que « $\neg$ ». On pourrait souligner visuellement cette intention en ajoutant des tirets: «il-n'est-pas-le-cas-que ». Il peut être éclairant ici de rappeler l'usage de deux grands logiciens dans leur prose informelle. Dans son essai de maturité, "Die Verneinung», Frege formule de la manière suivante la double négation de la vérité que la Schneekoppe est plus haute que le Brocken de la manière suivante ${ }^{24}$ :

(DN) Il n'est pas vrai que la Schneekoppe n'est pas plus haute que le Brocken.

Le signe de négation «inséré (eingefügt)» est (sémantiquement) atomique ${ }^{25}$, mais on ne pourrait pas exprimer la double négation de cette vérité géographique par le bégaiement non grammatical suivant: "la Schneekoppe n'est pas pas plus haute que le Brocken*. Si on place «ne... pas» devant une phrase niée de manière interne, le résultat n'est pas acceptable grammaticalement non plus. Comme solution d'urgence à ce problème grammatical ennuyeux, Frege préfixe un connecteur verbeux (un proche parent de «il n'est pas le cas que ») à cette phrase, avec la claire intention que ce connecteur fasse le même travail que le «ne... pas ${ }^{26}$ inséré dans la phrase. Voici ce qu'Alfred Tarski dit à propos de son propre usage du même connecteur pour la même tâche ${ }^{27}$ :

Pour des raisons stylistiques, nous employons parfois l'expression «il n'est pas vrai que » à la place du mot "ne » ( "nicht»). Par là, nous traitons cette expression complète comme un seul mot, sans assigner la moindre signification autonome ni à ses parties individuelles ni, en particulier, au mot «vrai» dont l'occurrence est présente en elle.

Frege aurait certainement endossé cette remarque. Dans son article "Gedankengefüge », il prend comme langue cible une version régimentée de l'allemand. Dans un français régimenté de manière semblable, $(\mathrm{DN})$ serait remplacé par $\left(\mathrm{DN}_{\mathrm{R}}\right)$ :

23. En cela, je suis parfaitement d'accord avec Napoli, 2006, p. 245.

24. Frege, 1919 , p. 148 (pagination originale).

25. Pour appuyer le caractère sémantiquement $e t$ syntaxiquement atomique du signe de négation inséré, on peut considérer ici une version en langue familière de (DN) : "Il est pas vrai que la Schneekoppe est pas plus haute que le Brocken ", où le morphème "ne " est effectivement omis, ou encore à une version soutenue de (DN): «Il $n$ 'est vrai que la Schneekoppe $n$ 'est plus haute que le Brocken. » Ces deux exemples montrent que la négation française peut aussi être syntaxiquement atomique et peut donc s'harmoniser avec les cas anglais («not») et allemand («nicht») discutés originellement par l'auteur (N.d.T.).

26. Ou qu'il fasse le même travail que le «ne» (version soutenue de (DN)), ou encore que le "pas" (version familière de (DN)) (N.d.T.).

27. Tarski, $1935, \mathbb{2}$, note 17 . Traduction tenant compte des deux N.d.T. précédentes (N.d.T.). 
$\left(\mathrm{DN}_{\mathrm{R}}\right)$ Non (non (La Schneekoppe est plus haute que le Brocken)).

Le connecteur de négation du français régimenté est simplement une variante typographique du petit trait vertical dans la Begriffsschrift de Frege ou du trait en crochet de Gentzen, par exemple ${ }^{28}$. Si le connecteur «Il-n'estpas-vrai-que » est insécable, alors sa compréhension ne pourra bénéficier de celle des mots dont il est ostensiblement constitué ${ }^{29}$. (Comprendre «mon» et «onde» ne nous aide nullement à comprendre «monde»). Il y a donc une vaste différence entre «il n'est pas vrai que » et sa contrepartie en tirets, et la même chose peut être dite pour «il n'est pas le cas que». Le sujet à discussion plus haut était cet opérateur. En ce qui concerne sa contrepartie en tirets, ou le crochet (ou le "pas» dans «la neige est pas bleue»), je ne me permets pas d'oser soutenir que c'est secondaire à toute construction prédicative.

\section{Des problèmes logico-sémantiques ou des bizarreries grammaticales?}

Mulligan soutient que des instances démaquillées de «que $p$ » dans des phrases de la forme «que $p$ est vrai », contrairement à des instances de «la proposition que $p$ » sont «difficilement» des termes singuliers «à part entière ${ }^{30}$. Il soutient sa thèse au moyen d'un argument par l'échec de la substitution. Très certainement, «la proposition que certaines chaussures neuves sont inconfortables est (identique à) la proposition que certains souliers neufs sont inconfortables" est grammaticalement casher, alors que *Que certaines chaussures neuves sont inconfortables est (identique à) que certains souliers neufs sont inconfortables* ne l'est pas ${ }^{31}$. Mais est-ce que cette observation justifie l'affirmation selon laquelle les subordonnées complétives démaquillées ne sont pas des termes singuliers décents? Dans «Wolfgang Künne est l'auteur de Conceptions of Truth», aucun des termes à droite ou à gauche du "est» d'identité ne peut être remplacé salva congruitate par un pronom de la première personne du singulier : est-ce que cela est censé montrer que, contrairement à ces deux termes, «je», prononcé par moi, ne désigne pas Wolfgang Künne? (Mme Anscombe a argumenté jadis de façon notoire en faveur de cette affirmation concernant ce pronom, mais son argument n'était pas basé sur un tic de la grammaire). Tout autant qu'il

28. Frege, 1923, orig. p. 41, et passim.

29. Il peut être expliqué comme un opérateur dont l'application à une phrase donne pour résultat une phrase qui contredit la phrase originale.

30. Cf. Mulligan, 2010, $\mathbb{4}$. Mulligan parle à cet endroit de "noms" plutôt que de «termes singuliers », mais il a aussi utilisé ailleurs la dernière expression dans le même type de contexte.

31. Deux de ses propres exemples ne sont pas «du français acceptable» pour une raison supplémentaire: il confondent les langues comme le fait *la capitale de la Grande-Bretagne est The capital of Great Britain*. (J'ai de bonnes raisons de soupçonner que l'auteur veut me faire marcher avec un de ses exemples surdéterminés. Voici donc mon commentaire sinistre: Tous les cols blancs criminels non Suisses évadés fiscaux apprécieront cet exemple. 
est nécessaire, pour insérer ce pronom dans le schéma " $a$ est identique à $a$ », d'avoir deux changements supplémentaires ${ }^{32}$, ces changements sont aussi nécessaires pour l'insertion d'une instance de "que $p$ ». Dans le dernier cas, nous devons préfixer deux fois une expression de la forme "le___" afin de se conformer aux exigences de la grammaire française. Nous devons distinguer la proposition qu'un décret a été émis par Jules César (qui peut être vraie) de l'état de choses qu'un décret a été émis par Jules César (qui peut être le cas), et nous devons aussi distinguer la proposition et l'état de choses de l'événement de l'émission d'un décret par Jules César (qui, selon les Évangiles, a été passé à un certain moment). Ainsi, dans le contexte "nu» "Que $p$ est que $p$ ", les subordonnées complétives nécessitent une désambiguïsation par apposition ${ }^{33}$.

Une instance de "que $p$ " peut-elle flanquer le «est " sur un côté de l'identité si, de l'autre côté, nous avons ce que Mulligan considère être un terme singulier "à part entière" ? Il ne se penche pas sur les cas où un nom propre occupe un des deux côtés, comme dans *Le logicisme est que l'arithmétique est réductible à la logique*. C'est très clairement non grammatical, comme tous les autres cas dans lesquels la place vide dans «... est que $p$ » est remplie par le nom propre d'une proposition. (Il n'y a pas tant de noms de ce genre, et la plupart d'entre eux peuvent être trouvés dans les manuels de philosophie.) Ici encore, on peut rétablir la loi et l'ordre grammatical en ajoutant un préfixe: «Le logicisme est le que l'arithmétique est réductible à la logique.» Cette observation montre-t-elle que des instances de «que $p$ » ne sont pas des termes singuliers à part entière? Je ne pense pas. Cela montre seulement que dans certaines positions ils ont besoin d'une béquille. Une comparaison peut être utile ici. Contrairement à "Johannes Brahms », la description définie co-désignative «l'ami de Clara Schumann » a besoin d'un certain ajout avant de pouvoir être insérée dans la place vide dans «Le compositeur hambourgeois __ était très admiré à Vienne » (sc. "qui était l'ami de Clara Schumann»). Personne ne considérerait cela comme une preuve que les descriptions définies ne sont pas des termes singuliers décents.

Mulligan considère les cas où une description définie remplit la place vide dans une instance de "...est que $p$ ». Un de ses exemples est la phrase grammaticalement impeccable «La proposition préférée de Casimir Lewy

32. Dans une version régimentée du français, "Hespérus est Phosphorus", "Je suis Gustav Lauben », «Hespérus est Hespérus » et "Je suis identique à moi-même » devraient être reformulées de telle manière qu'elle deviennent toutes des instances du schéma « $=(x, y)$ », et les reformulations pourraient être traitées comme des représentations de la structure fondamentale des expressions originales.

33. Mulligan fait un pas de trop dans ce passage irénique lorsqu'il suggère que les subordonnées complétives nécessitent toujours une désambiguïsation. Lorsqu'elles sont enchâssées dans «( ) est vrai», «( ) est le cas» ou «( ) s'est produit au temps t », par exemple, aucune désambiguïsation n'est nécessaire. 
est qu'une renarde est la femelle d'un renard.» Il affirme: «Si la copule [? ${ }^{34}$ ] exprime ici l'identité, la phrase est alors elliptique de «La proposition préférée de Casimir Lewy est la proposition qu'une renarde est la femelle d'un renard." Quelqu'un qui considère les subordonnées complétives comme des termes singuliers authentiques ne verra pas la nécessité d'une telle manœuvre. Son deuxième exemple pour ces types de cas est une phrase comme

[B] La croyance de Ben est qu'un jour, tous les morts ressusciteront (brièvement : que $R$ )

Je dirais que c'est là un cas dégénéré de la forme "...est que $p$ ", car cela exprime la même proposition que «Ben croit que $R$ ». Mais j'aimerais discuter le commentaire de Mulligan sur [B]. Si on lit [B] comme un énoncé d'identité et qu'on considère la subordonnée complétive comme désignant une proposition, alors, affirme-t-il, [B] exprimerait une fausseté. Mais pourquoi ? "Les propositions n'ont aucune des propriétés intrinsèques qu'ont les états ou les dispositions psychologiques.» Ce diagnostic est correct seulement si «la croyance de Ben» dans [B] désigne un état ou une disposition psychologique. Or supposons que Ben et Anne croient que $R$. Si nous disons que la croyance de Ben que $R$ est due à l'endoctrinement de sa jeunesse, alors que la croyance que $R$ d'Anne est le résultat d'une conversion à l'âge adulte, nous n'assignons pas deux différentes origines à un seul et même item, et si nous disons que la croyance que $R$ de Ben est ferme et que la croyance d'Anne est aisément mise à mal, on ne dit pas de la même chose qu'elle est à la fois ferme et mise à mal. Dans de tels énoncés, nous parlons d'une croyance comme quelque chose dont l'identité est dépendante d'agents croyants. Appelons cela un état de croyance. Lorsqu'Anne change d'idée eu égard à la question de savoir si $R$ alors que Ben demeure obstiné, un seul des deux états de croyance persiste. Mais la croyance de Ben telle qu'elle est caractérisée dans [B] peut aussi être la croyance d'Anne. Ainsi, l'expression «la croyance de Ben» dans $[\mathrm{B}]$ ne désigne pas une croyance mais plutôt ce que Ben croit - le contenu d'un des états de croyance de Ben qui peut aussi être le contenu d'un des états de croyance d'Anne. Ce contenu n'a en effet "aucune des propriétés qu'ont les états et les dispositions psychologiques ", car c'est une proposition (Conceptions of Truth, p. 250). Ainsi, [B] peut très bien servir à faire un énoncé d'identité (dégénéré) à propos d'une proposition: selon [B], la proposition que $R$ est identique au contenu d'un des états de croyance de Ben.

34. C'est peut-être uniquement mon orientation frégéenne qui me pousse à penser la copule comme un opérateur formant des prédicats sur des termes généraux: compris de cette manière, le «est» de l'identité n'est pas une copule mais un prédicat diadique (Künne, 2006, p. 249-253). 
Mulligan affirme que toutes les instances de «Que $p$ est vrai» sont «moins que des phrases à part entière ». La preuve qu'il apporte pour cette affirmation inamicale est un point intéressant à propos des restrictions sur l'enchâssement que je n'avais jamais noté auparavant. Des séquences comme *je me demande si que la neige est blanche est vrai* et "si que la neige est blanche est vrai alors la neige est blanche* sont inacceptables grammaticalement, alors que leur contreparties en «il est vrai que » sont correctes. Très bien (si son oreille et celle de ses témoins sont fiables). Mais alors, je pense que "je me demande si deux âmes, hélas! résident en mon sein* et "si malheureusement deux âmes résident en mon sein, je ferais mieux de consulter mon psychiatre* sont grammaticalement inacceptables, alors que leurs contreparties innocentes sont acceptables ${ }^{35}$. Cela montre-t-il que (des instances de) « $p$, hélas» et «malheureusment, $p$ » ne sont pas des phrases à part entière et que, pace Frege $^{36}$, elles n'expriment pas la même proposition que " $p$ » tout court? Ou encore, comparons la curieuse séquence *Si si Socrate était sage quelqu'un était sage, alors il y a au moins une vérité nécessaire* avec sa contrepartie casher (espérons qu'elle le soit): "Si quelqu'un était sage si Socrate était sage, alors, etc.» Ce contraste montre-t-il que des instances de «si $p, q$ » ne sont pas des "phrases à part entière » alors que des instances de « $q$, si $p$ » le sont? Cela montre-t-il que (pace tout le monde) deux conditionnelles qui sont liées de telle manière n'expriment pas la même proposition? Il semble que l'argument de Mulligan en prouve trop.

Mulligan lui-même semble un peu effarouché ( «malheureusement...») par une autre conséquence de son argument de l'enchâssement. Il s'avère qu'aucun schéma commençant par «Si que $p$ est vrai» n'a d'instance de substitution grammaticalement acceptable, et donc aucune n'a une instance qui exprime une vérité. Il est à juste titre décontenancé par cela, car endosser de tels schémas est partie intégrante de sa propre conception telle que caractérisée plus haut par (5), (7) et (9). Mais pourquoi ne pas considérer tous ces schémas comme étant formulés dans des versions régimentées du français permettant de tels enchâssements? On peut le signaler par une minuscule intervention: «Si: que $p$ est vrai, alors: ..."

Ce ne sont pas toutes les caractéristiques grammaticales des langues vernaculaires qui sont importantes pour élucider le concept de vérité. En français, toutes les instances de substitution du schéma " $p$ ssi il est vrai que $p$ » sont grammaticalement bien formées. Mais, malheureusement, la traduction allemande de ce schéma n'a pas d'instance de substitution grammaticalement acceptable dans laquelle le prédicat n'est pas un verbe complet. Si on observe attentivement:

(W) Schnee ist weiß gdw, es wahr ist, dass Schnee weiß ist,

35. Cf. Goethe, Faust I. (N.d.T.).

36. Cf. Frege. 1918, orig. p. 63 (N.d.T.). 
on note que ce n'est pas vraiment une instance du schéma. Dans le cas du latin classique, il n'est même pas nécessaire de regarder attentivement. La traduction de "s'il est vrai que la neige est blanche, alors la neige est blanche" est "Si nivem albam esse verum est, nix alba est", où on a une construction en accusatif-cum-infinitif plutôt qu'une phrase enchâssée dans l'antécédent. Même si j'aime ma langue maternelle, je sympathise avec ceux parmi mes lecteurs qui s'exclameront sur ce point: "Tant pis pour l'allemand (le latin)! » Dans une version régimentée de l'allemand, (W) est transformé par

\section{$\left(\mathrm{W}_{\mathrm{R}}\right)$ Schnee ist weiß gdw. es ist wahr, dass Schnee ist weiß.}

Si quelqu'un voulait se plaindre en disant, «ce n'est pas grammaticalement acceptable en allemand», je lui répondrais «d'accord, mais je n'ai jamais prétendu que ce l'était ». Frege a explicitement noté le même genre de problème, si on peut parler ainsi, dans son fragment "Logische Allgemeinbeit». Lorsqu'on instancie le schéma d'argument «p. Wenn p, dann q. Also $q$ ", la deuxième prémisse sera rarement grammaticalement acceptable: *Wenn Napoleon ist ein Mensch, dann Napoleon ist sterblich*. Frege était tout à fait conscient du fait — et à juste titre ne s'en laissa point troubler - que les règles syntaxiquement précises de dérivation sont seulement applicables à une version régimentée des langues vernaculaires ${ }^{37}$.

Certains échecs de substitutivité ne sont certainement pas simplement des tics de la grammaire, mais, tout aussi certainement, certains le sont. J'aimerais pouvoir offrir ici un critère qui nous permette de les départager, mais hélas! je n'ai pas de tel critère à ma disposition.

\section{Le connecteur de vérité est-il insécable?}

Prima facie, la réponse de Mulligan à cette question semble lamentable. Après tout, la présence de «vrai " dans «il est vrai que $p$ " ne semble pas être un accident orthographique comme sa présence dans "livraison" (Conceptions of Truth, p. 351). Mais le cas des expressions idiomatiques montre que le fait que notre connecteur de vérité semble structuré ne fait pas grand effet ici. Prenez la notice nécrologique argotique «Ben a cassé sa pipe»: le verbe «a cassé » n'intervient pas ici comme un prédicat à deux places, et le syntagme «sa pipe » n'intervient pas ici non plus comme terme singulier, car on ne peut raisonnablement conclure en partant de ce rude énoncé qu'il y a quelque chose que Ben a cassé, et on ne peut non plus se demander de manière sensée si la pipe qu'il a cassée est la même que la pipe que Tom a cassée la veille. Ainsi, contrairement au prédicat dans «Anne a cassé sa tasse ", le prédicat dans la notice nécrologique est structuré de manière uniquement ostensible, et de ce point de vue il ne diffère qu'en apparence du prédicat dans "Ben est décédé». De la même façon, selon la conception de 
Mulligan, ce qui précède « $p$ » dans «il est vrai que $p$ » est structuré de manière uniquement ostensible. Mais notons la différence éclatante entre l'idiome et le connecteur de vérité : alors que "casser sa pipe », comme n'importe quelle autre expression idiomatique, jouit de sa propre notice dans tout bon dictionnaire français, le connecteur de vérité n'a quant à lui jamais été vu digne d'une telle notice, à ma connaissance. Je crois savoir pourquoi. C'est parce que le connecteur de vérité est vraiment structuré. La compréhension d'une expression idiomatique n'est jamais ou rarement favorisée par la saisie du sens des mots dont elle est ostensiblement constituée, et la compréhension de "vrai » n'a de toute évidence aucun rapport avec la compréhension de «livraison». Donc, si le connecteur de vérité est insécable, alors sa compréhension ne peut bénéficier de la compréhension d'un mot qu'il contient ostensiblement. N'est-ce pas là un cas pour appliquer le modus tollens? Si on apprend à se débrouiller avec «vrai » dans des contextes prédicatifs comme "c'est vrai» et "son hypothèse est vraie", ne peut-on pas se baser là-dessus lorsqu'on rencontre le connecteur de vérité pour la première fois?

De plus, la construction «il n'est pas vrai que » ne nécessite-t-elle pas d'attribuer une structure interne au connecteur de vérité? Mulligan serait tenté de répondre non (comme il le fait dans des cas structurellement semblables), parce que la construction a la structure fondamentale de «Il n'est pas le cas qu'il est vrai que ». Mais cette stratégie nous ramène à la question de savoir si cet opérateur préfixé est insécable. J'ai soutenu qu'il ne l'était pas. La phrase logico-française « $\neg$ (il est vrai que $p$ )», d'autre part, n'est structurellement isomorphique avec aucune phrase du français, car (comme je l'ai déjà dit) le français n'a aucun connecteur à un seul mot servant à contredire et qui peut être préfixé à une phrase. Le «ne... pas» inséré dans des phrases comme «Ernest n'est pas fatigué » ressemble beaucoup à un opérateur formant des prédicats et appliqué à des prédicats ${ }^{38}$, et si cette apparence n'est pas trompeuse, la construction «il n'est pas vrai que» fournit une preuve supplémentaire à ma thèse selon laquelle l'opérateur de vérité contient le prédicat de vérité.

Mulligan essaie d'affaiblir la résistance contre sa thèse d'insécabilité en comparant le connecteur de vérité avec les connecteurs qui ont des contreparties en un mot, comme des adverbes ou des adverbes de phrases. Il se demande si les instances correspondantes de «il est probable (possible, ...) que $p$ » et «Probablement (possiblement, ...) $p$ » veulent dire la même chose. Sa question rhétorique mérite le signe d'assentiment qu'elle est censée sus-

38. En italien, la négation est placée devant le prédicat ("Ernesto non è stanco", «Ernest non dorme», en allemand, elle est parfois placée après le prédicat ( Ernst schläft nicht»). 
citer $^{39}$. Puisqu'il n'y a pas de telle version condensée du connecteur de vérité en français (ou en anglais, en allemand, etc. ${ }^{40}$, Mulligan en invente $u^{41}$. En français-plus, il y a un adverbe de phrase en un seul mot qui est lié à notre connecteur de vérité de la même manière que "probablement" est lié à «il est probable que». Il suggère que des constructions avec le connecteur de vérité français sont «dérivées de » constructions avec l'adverbe de phrase en français-plus, c'est-à-dire de constructions avec un mot qui n'existe pas en français. Je dois avouer que je trouve cette suggestion tout simplement déroutante. Mais, de toute façon, l'insécabilité d'un adverbe de phrase en un seul mot qui est (supposément) synonyme avec un connecteur verbeux offret-elle vraiment un support à la thèse que ce dernier est lui aussi insécable? "Ermelyn est un renard femelle» et «Ermelyn est une renarde » disent aussi la même chose, mais personne n'en conclurait que le prédicat dans la phrase plus longue est insécable. De plus, deux phrases qui sont distinctes seulement à une certaine position peuvent avoir la même signification, même si les expressions à cette position ne sont pas synonymes ${ }^{42}$. (Prenons "Socrate défendit sa personne» et "Socrate défendit Socrate»). Seuls les constituants authentiques (et co-catégoriels) de phrases peuvent être des synonymes. «Probablement» est un adverbe de phrase et, comme tel, c'est un constituant authentique d'instances de «Probablement, $p$ ». Si «il est vrai que » est un fragment de phrase hybride constitué d'un explétif, un prédicat et une conjonction de subordination, alors ce n'est pas un constituant authentique d'instances de «il est vrai que $p$ ». Je crois que nous devrions affirmer l'antécédent. Une raison de le considérer comme un tel hybride se base sur l'argument de la parenthèse que j'ai utilisé plus haut pour montrer que «il n'est pas le cas que » est un hybride, et que Kent Wilson ${ }^{43}$ a utilisé dans le présent contexte: nous pouvons insérer une expression entre parenthèses, comme dans «il est vrai, comme nous le savons tous, que $p$ »; ainsi, «que» se joint à la phrase suivante pour former une subordonnée complétive. Mulligan

39. Prenons note que, parfois, un connecteur («il appert que») qui peut être condensé en un adverbe de phrase d'un seul mot («apparemment») n'a pas de contrepartie prédicative.

40. Comme le note Mulligan, les adverbes anglais «truly» ("He said truly that $\mathrm{p}$ ») et «verily» ("Verily I say unto thee») ne sont pas dans la même branche, tout comme "wahrlich» en allemand. Incidemment, "Er ist wahrlich [ou: wahrhaftig] ein guter Philosoph» ne porte aucune connotation de sincérité ou de véridicité - cela exprime la même idée que «il est vraiment un bon philosophe». Cf. Bolzano, 1837, vol. I, p. 123. En ce qui concerne la question de Mulligan à propos d'un «idiome allemand »: "Wahr ist, das p » n'est qu'une variante stylistique de «Dass p, ist wahr». Un exemple schématique: "Wahr ist, dass p, aber dass $\mathrm{q}$, ist keineswegs wahr».

41. Les cas anglais discutés par Mulligan 2010 ont été adaptés au français (N.d.T.).

42. Cf. Geach, 1968, p. 61 sur la «cancelling-out fallacy».

43. Wilson, 1990, p. 23-24 (cité par Mulligan et mentionné dans un autre contexte dans Conceptions of Truth, p. 69). 
admet que c'est un «solide argument » en faveur de ma thèse ${ }^{44}$, et je ne pense pas que sa "réponse partielle» porte beaucoup de conviction.

Dans Conceptions of Truth, j'ai affirmé qu'un certain usage anaphorique de "aussi » nous fournit une preuve pour la thèse que «il est vrai que $p »$ contient une occurrence authentique du prédicat de vérité (et donc une preuve contre la thèse que le connecteur de vérité est insécable). En commentant une dispute entre Ben et Anne à propos de leur superviseur, quelqu'un pourrait dire:

(A) Il est vrai qu'il est brillant, mais sa remarque critique à son endroit est aussi vraie.

Le rôle d' "aussi» en (A) peut être expliqué, comme je l'ai écrit, si l'usage du prédicat de vérité dans la seconde moitié de (A) est précédé par un autre usage de ce même prédicat dans la première moitié. Mulligan essaie de montrer qu'il y a une autre explication, mais il concède que la mienne est « indubitablement plus simple» que la sienne. Sa propre explication, dont il croit qu'elle a un domaine d'application plus large, est basée sur l'assomption que «quiconque comprend la première moitié de $(\mathrm{A})$ sait qu'il est vrai que N.N. est brillant ssi la proposition qu'il est brillant est vraie». (J'ai modifié minimalement l'exemple.) Mais Mulligan n'avait-il pas rejeté plus tôt ce genre d'assomption, et à juste titre, pour la raison que comprendre "la proposition que $p$ est vraie" est conceptuellement plus exigeant que comprendre «il est vrai que $p$ »? Peu importe, je ne suis plus satisfait par l'argument du «aussi». La raison de mon insatisfaction est assez simple; considérons une variante de $(\mathrm{A})$ :

$\left(\mathrm{A}^{*}\right)$ Il est en effet brillant, mais sa remarque critique à son endroit est aussi vraie.

Il est certain que la cohérence de $\left(\mathrm{A}^{*}\right)$ peut être explicable sans réaliser la prouesse impossible de trouver une autre application au prédicat «est vrai » dans la première partie de la conjonction ${ }^{45}$. Ainsi, la cohérence de $(\mathrm{A})$ ne peut dépendre non plus de la disponibilité de l'explication, et cette explication n'offre aucun support à la thèse que le prédicat «( ) est vrai» est présent dans «il est vrai que $p »$.

44. Cf. Mulligan, 2010, p. 577. Il y a un autre argument wilsonien (loc. cit.) qui doit être noté. La manière par laquelle on répartit le ballast en partant de "Certaines personnes refusent d'admettre qu'elle est corrompue, mais il est vrai qu'elle est corrompue " vers "Certaines personnes refusent d'admettre qu'elle est corrompue, mais c'est vrai " montre que "que $p$ » est un constituant authentique de «il est vrai que $p$ ». (Si le mot "que» était enchaîné à "vrai ", il devrait être possible de terminer par «il est vrai que», mais la grammaire ne nous y autorise pas.)

45. Ou encore, prenons: «Lorsqu'il quitta Londres, il pleuvait des cordes, mais lorsqu'il arriva à Rome, la température était mauvaise aussi. » Il n’y a pas de prédication de "mauvaise » dans la première partie, mais pourtant l'anaphore est compréhensible. 
On obtient un meilleur argument en faveur de ma liste de priorités si on élargit la conception. Mulligan se concentre sur des phrases comme $(\mathrm{CV})$, $(\mathrm{PV})$ et $(\mathrm{PV}+)$ et il offre une conception provoquante de leurs interrelations. Il n'y a pas un seul exemple d'un usage de "vrai» qui ne contienne pas un «que» suivi d'une phrase. Du début à la fin, son article porte sur les façons expressives de parler de la vérité, c'est-à-dire sur les phrases dans lesquelles une locution de vérité est appliquée à quelque chose qui exprime - ou contient une partie qui, elle, exprime - un candidat à la véritét ${ }^{46}$. Bien sûr, ce n'est pas en soi une raison de se plaindre. C'est seulement lorsqu'on lit le tout dernier paragraphe de son article qu'on commence à soupçonner que son régime d'exemple est peut-être un peu trop monotone.

En ce qui concerne les manières expressives de parler de la vérité, les théoriciens de la redondance ont raison sur un point: ce que nous disons est fortement équivalent avec quelque chose que nous pourrions dire sans employer de locution de vérité. Mais une élucidation de notre concept de vérité ne peut se permettre de mettre de côté des manières de parler de la vérité qui sont non expressives, comme:

- Tout ce qui découle d'une vérité est vrai.

- Tout ce que le pape dit ex cathedra est vrai.

- Le dogme de l'infaillibilité papale est vrai.

- Son hypothèse préférée est vraie.

- Le faillibilisme est vrai.

Aucun parsage de phrases de ce genre pourra mettre en lumière un connecteur de vérité - eu égard à ce genre de manière de parler de la vérité, le parsage prédicatif est obligatoire. Or une élucidation de notre concept de vérité ne peut se permettre une vision en tunnel. Il doit prendre en considération les manières expressives et non expressives de parler de la vérité, et il doit faire la lumière sur leur interaction à l'intérieur d'un seul et même argument. Un opérateur servant à former des phrases et appliqué sur des phrases ne peut possiblement avoir le même sens qu'un prédicat, et si le connecteur de vérité est insécable, alors il ne contient aucune composante authentique qui a le même sens que le terme général transformé par la copule en prédicat de véritét ${ }^{47}$. Conséquemment, si on suit la piste de Mulligan sur le connecteur de vérité, il sera extrêmement difficile, c'est le moins qu'on puisse dire, d'expliquer l'impression irrésistible que l'argument suivant est formellement valide:

46. Cf. Conceptions of Truth, p. 52 et passim sur des manières de parler de la vérité qui sont "propositionally revealing" par rapport à "propositionally unrevealing».

47. Le prédicat de vérité n'est pas insécable non plus. Les arguments de Kent Wilson peuvent être aisément adaptés afin de montrer que les termes généraux sont des constituants authentiques des prédicats. L'insertion d'une parenthèse est possible: «La proposition favorite de Casimir Lewy est — comme nous le savons tous — vraie »; tout comme le déshabillage (au moins en anglais): "Not every statement in his book is true, but this one is < true $>$." 
(P1) Il est vrai que tout nombre pair plus grand que 2 est la somme de deux nombres premiers.

(P2) Que tout nombre pair, etc., est la conjecture de Goldbach, donc

(C)La conjecture de Goldbach est vraie.

Dans la compréhension de (P1) par Mulligan, cet argument devrait être classé comme enthymématique. Par contraste, il est très facile d'expliquer l'impression de validité formelle si on autorise le parsage prédicatif de (P1) et qu'on considère (P2) comme un énoncé d'identité, puisqu'alors l'impression est véridique: l'argument exemplifie un schéma qui est universellement valide dans le calcul des prédicats, sc. $F a, a=b \backslash F b$. On ne peut rendre compte de manière adéquate des nombreux aspects de notre usage de «vrai » sans faire intervenir des vérités dans le portrait, c'est-à-dire des choses auxquelles s'applique le prédicat de vérité.

Enfin, il y a au moins une affirmation substantielle en philosophie de la logique qui dépend de la présence de prédicats de vérité dans des instances de «il est vrai que $p$ ». Des philosophes comme Frege et Strawson soutiennent que il est ni vrai ni faux que, disons (la planète) Vulcain est plus grande que la Lune, puisque le terme singulier "Vulcain» ne désigne rien. C'est une question substantielle de savoir s'ils ont raison ou non, mais le problème disparaîtrait si le parsage en connecteur de vérité de «il est vrai que $p$ » était le seul autorisé. Supposons que «P» soit l'abréviation d'une phrase déclarative qui ne dit rien de vrai ou de faux. Dans ce cas, «il-estvrai-que $\mathrm{P}$ » n’exprimerait pas non plus une vérité ou une fausseté, car si le connecteur de vérité est insécable (comme le signale l'emploi des tirets), le thème de la phrase avec le préfixe est alors le même que celui de la phrase enchâssée. La même chose est valable en ce qui concerne "il-est-faux-que $\mathrm{P} »$, car cela exprime une vérité (fausseté) seulement dans la mesure où «non-P» le fait, et si ce que « $\mathrm{P}$ » affirme tombe dans un fossé de valeur de vérité, alors ce que «non-P» affirme doit faire de même. Conséquemment, «Non (il-est-vrai-que P) et non (il-est-faux-que P)» échouent également à affirmer quelque chose de vrai ou de faux. Mais les défenseurs du fossé de valeur de vérité veulent affirmer une vérité lorsqu'ils disent «il n'est ni vrai ni faux que Vulcain est plus grande que la Lune". C'est seulement dans le parsage prédicatif de "il est vrai (faux) que $\mathrm{P}$ » qu'un tel énoncé a une chance d'être vrai, car alors, le thème de « il est vrai (faux) que $\mathrm{P}$ » est la proposition que P. En bloquant obstinément le parsage prédicatif, l'adversaire de la thèse du fossé gagnerait beaucoup trop facilement une victoire sur Frege et Strawson. 
Ainsi, tout bien considéré, la «cible de la plupart des théories de la vérité, y compris celle de Künne ${ }^{48}$ ", ne semble pas avoir été faussement «identifiée»: c'est le prédicat de vérité qui porte la culotte ${ }^{49}$.

Traduit de l'anglais par Guillaume Fréchette

\section{Bibliographie}

Bolzano, Bernard. Wissenschaftslehre, 4 vol., Sulzbach, 1837.

Frege, Gottlob. 1891. "Function und Begriff», reproduit avec la pagination originale in Funktion - Begriff - Bedeutung, M. Textor (ed.), Göttingen, 2002.

1918. «Die Verneinung»,

1923. "Gedankengefüge »,

. 1923-1924. "Logische Allgemeinheit », tous reproduits avec la pagination originale et un commentaire, in Künne, 2010.

Geach, Peter Thomas. Reference and Generality, édition révisée, N. Y., Ithaca, 1968.

- Logic Matters, Oxford, 1972.

Kretzmann, Norman. "Medieval Logicians on the Meaning of the propositio ", in Journal of Philosophy, 67, 1970, 767-787.

Künne, Wolfgang. 1983. Abstrakte Gegenstände - Semantik und Ontologie, Francfort/M, ${ }^{2} 2007$.

- Conceptions of Truth, Oxford, 2003.

- "Properties in Abundance", in Universals, Concepts and Qualities, P. F. Strawson \& A. Chakrabarti (eds.), Ashgate, 2006, 249-300.

—. "A Dilemma in Frege's Philosophy of Thought and Language ", in Rivista di estetica 34, FS for Diego Marconi, 2007, 95-102.

- "Analyticity and Logical Truth: From Bolzano to Quine», in Versuche über Bolzano / Essays on Bolzano, Sankt Augustin, 2008, 233-303.

- Die Philosophische Logik Gottlob Freges, Francfort/M, 2010.

Mulligan, Kevin. "The Truth-Predicate vs the Truth-Connective. On Taking Connectives Seriously ", in Dialectica, vol. 64, no 4, 2010, 565-584.

Napoli, Ernesto. «Negation", in Grazer Philosophische Studien 72, 2006, 233-252.

Prior, Arthur N. "Correspondence Theory of Truth ", in The Encyclopedia of Philosophy, vol. 2, P. Edwards (ed.), Londres, 1967, 223-232.

- Objects of Thought, Oxford, 1971.

Schnieder, Benjamin. "Truthmaking Without Truthmakers », in Synthese 152, 2006, 21-46.

—_. "A Puzzle About "Because" ", à paraître-1, dans un numéro spécial de Logique et Analyse.

—. "The Asymmetry of "Because" ", à paraître-2.

48. Cf. Mulligan, 2010, p. 583 (N.d.T.).

49. Je remercie Paul Boghossian et Kevin Mulligan pour avoir attiré mon attention sur différents aspects de mon travail sur le concept de vérité qui requièrent des clarifications, des défenses supplémentaires, des développements substantiels, ou qui doivent simplement être refusés. Je remercie également Ian Rumfitt pour ses encouragements aux moments et aux endroits précis où ils ont été nécessaires. Merci également à Anne Desler, Christian Folde, Maria Kuper, Kevin Mulligan, Pawel Rasch, Alexander Steinberg et Maik Sühr pour leur aide à la préparation de la révision finale de cette réplique. 
Tarski, Alfred. 1935. "Der Wahrheitsbegriff in den formalisierten Sprachen », in Tarski, Collected Papers, vol. II, Bâle, 1986, 51-198.

Wilson, W. Kent. "Some Reflections on the Prosentential Theory of Truth ", in Truth and Consequences, M. J. Dunn \& A. Gupta (eds.), Dordrecht, 1990, 19-32. 